\title{
Progress in the Molecular Genetics of Hypertrophic Cardiomyopathy: A Mini-Review
}

\author{
Tao Tian Yaxin Liu Xianliang Zhou Lei Song \\ Department of Cardiology, State Key Laboratory of Cardiovascular Disease, Fuwai Hospital, National Center for \\ Cardiovascular Disease, Chinese Academy of Medical Sciences and Peking Union Medical College, Beijing, China
}

\section{Key Words}

Hypertrophic cardiomyopathy · Gene mutation .

Pathogenesis · Modifier · Aging · Genetic diagnosis

\begin{abstract}
Background: Hypertrophic cardiomyopathy (HCM), which is characterized by unexplained and asymmetric left ventricular hypertrophy in the absence of other cardiac or systemic diseases, is an inherited cardiovascular disease and presents rising penetrance with aging. Objective: The purpose of this review is to offer an outline of recent progress in the molecular genetics of HCM and to discuss characteristics of elderly HCM patients. Methods: Studies were analyzed which included disease genes related to $\mathrm{HCM}$, relationships between genotype and phenotype, potential pathogenesis of HCM, and the features of elderly patients with HCM. Results: HCM is caused by mutations in genes encoding myofilament proteins of the sarcomere, Z-disc proteins, $\mathrm{Ca}^{2+}$-handling proteins, and other proteins related to the sarcomere. Phenotypic manifestations of HCM are not just determined by these genes; modifying genes and epigenetic factors also contribute to the complexity of the HCM phenotype. The potential pathogenesis of HCM involves dominant negative function, an imbalance of myocardial energetic metabolism, and haploinsufficiency. Late-onset HCM presents its own features in the distribution of causal genes. Mutations in
\end{abstract}

MYBPC3 may be the most common cause of delayed expression of $\mathrm{HCM}$, and the sarcomere gene screen is most likely to be negative in elderly HCM patients. Conclusions: Despite progress in the identification of genetic causes and pathogenesis of HCM, there are still some questions that need to be better understood. It remains a great challenge to identify the cause of $50 \%$ of HCM cases in patients without an identified mutation. The application of a new genetic study technology may completely uncover the genetic background of these cases. In addition, the influences of causal mutations on the function and signaling of cardiocytes are expected to be elucidated further.

Copyright $\odot 2013$ S. Karger AG, Basel

\section{Introduction}

Hypertrophic cardiomyopathy (HCM) is a mendelian disorder that is characterized by unexplained and asymmetric left ventricular (LV) hypertrophy and presents increased penetrance with aging. It is familial in approximately half of the cases and sporadic in the other cases. The epidemiology of HCM suggests that it is present in about 1 in 500 adults. Since 1990, when it was first dem-

T. Tian and Y. Liu contributed equally to this work.

\section{KARGER}

Fax +4161306 1234

E-Mail karger@karger.com

www.karger.com (c) 2013 S. Karger AG, Basel

0304-324X/13/0593-0199\$38.00/0

Accessible online at:

www.karger.com/ger
Prof. Xianliang Zhou and Prof. Lei Song

Department of Cardiology, State Key Laboratory of Cardiovascular Disease, Fuwai Hospital, National Center for Cardiovascular Disease, Chinese Academy of Medical Sciences and Peking Union Medical College, No. 167, Beilishi Road, Beijing 100037 (China) E-Mail zhouxianliang0326@hotmail.com and lsongqd@yahoo.com 
onstrated that a mutation in $\beta$-myosin heavy chain (MYH7) was responsible for HCM, more than 1,400 distinct mutations have been identified [1]. Most of these mutations are in genes that encode myofilaments of sarcomeres, Z-disc proteins, and $\mathrm{Ca}^{2+}$-handling proteins; some of these mutations have strong evidence for pathogenicity, and others have less evidence [2]. Of these mutations, $M Y H 7$ and MYBPC 3 account for most of the cases $[3,4]$. About $50 \%$ of HCM cases cannot be traced to a specific genetic defect by current genetic approaches, even though new mutations have been identified successively [1]. The phenotype of HCM is affected by disease genes as well as modifiers. Although the detailed pathogenesis remains incompletely understood, potential mechanisms involved in HCM have been proposed, including dominant negative function, haploinsufficiency, and an imbalance of myocardial energy metabolism.

In the past two decades, progress in molecular genetics has afforded us insights into the pathogenicity and pathogenesis of HCM. The purpose of this review is to offer an outline of recent advances about this mendelian disorder, including which genes are associated with HCM, how these mutations result in the phenotype, and what the relationship between genotype and phenotype is. Moreover, considering that HCM exhibits age-related penetrance and that there are few reviews about the elderly suffering from HCM, we will discuss the features of the elderly with HCM.

\section{Physiopathological, Pathological, and Clinical Characteristics of HCM}

HCM is defined as unexplained LV hypertrophy (especially asymmetric) without an apparent cause (i.e. severe hypertension, aortic stenosis, or systemic diseases that might cause LV hypertrophy). Apart from LV hypertrophy, due to mitral valve systolic anterior motion and mitral-septal contact, LV outflow tract obstruction occurs in approximately $70 \%$ of HCM cases at rest and/or with physiologic provocation [5]. Histologically, HCM is characterized by hypertrophy and disarray of cardiomyocytes and interstitial fibrosis of the myocardium. Its clinical manifestations are markedly heterogeneous, ranging from an asymptomatic lifelong course to heart failure, stroke, and sudden cardiac death (SCD). HCM is usually diagnosed by a maximal LV wall thickness $\geq 15$ $\mathrm{mm}$ through cardiac imaging; currently, this is commonly done with two-dimensional echocardiography and is increasingly done with cardiac magnetic resonance [6].

\section{Genetic Causes}

A cardiomyocyte is composed of numerous fine strands called myocardial myofibrils. Each myofibril is primarily composed of two kinds of filaments - termed thick and thin - that are organized into repeated subunits. These subunits are the sarcomeres. The Z-disc, which forms the borders of the sarcomere, lies between sarcomeres.

\section{Thick Filament}

Thick filaments are mainly made up of myosin and myosin binding protein C (MYBPC3). Two $\alpha$ - or $\beta$-myosin heavy chains (MYH6 or MYH7) construct myosin as a 'molecular motor' of contraction. The heavy chains dimerize and form a coiled-coil helix that makes up the myosin 'rod'. Each heavy chain unwinds from its accompanier and folds into the catalytic myosin 'head', generating a cross-bridge. The myosin 'neck' is located between the 'rod' and the 'head' segments. At the C-terminus of the myosin 'head', each heavy chain forms a lever arm domain, where one regulatory light chain and one essential light chain (MYL2 and MYL3) are combined. MYB$P C 3$ is located on each side of the A-band. It is not only anchored to the myosin 'rod' and titin by its C-terminus but also bound to the myosin 'neck' by the domain near the $\mathrm{N}$-terminus. Besides, its $\mathrm{N}$-terminus can interact with actin, which could contribute to the effects of $M Y B P C 3$ to increase cross-bridge cycling.

Mutations in $M Y H 7$ and MYBPC3 account for 30-40\% of all HCM cases [1, 3, 7]. Most of the mutations in MYH7 are missense mutations, and they cluster between exons 3 and 23, which encode the myosin 'head' and 'neck' [8]. In animal models, some of the MYH6 mutations give rise to increased maximal actin-activated ATPase activity and actin-sliding velocity, and to disrupting actin-myosin interactions. These changes may not only affect myofibril morphology but also alter ventricular performance, contributing to a compensatory hypertrophy. Approximately two-thirds of MYBPC3 mutations, including splice site, insertion/deletion, and nonsense mutations, are predicted to generate a truncated $\mathrm{mRNA}$ and protein. These mutant products can be degraded by nonsense-mediated mRNA decay and the ubiquitin-proteasome system, which results in an insufficient amount of normal full-length $M Y B P C 3$ and altered sarcomeric function.

\section{Thin Filament}

Thin filaments are composed of actin, tropomyosin, and troponin complex. Actin (ACTC1) is the backbone of the thin filament. The interaction between actin and my- 
osin is predominantly regulated by the troponin complex, which includes troponin $\mathrm{T}, \mathrm{I}$, and C (TNNT2, TNNI3, and TNNC1). In the absence of $\mathrm{Ca}^{2+}$, troponin I inhibits the binding of actin to myosin by interacting with actin-tropomyosin and fixing the troponin complex on actin through an interaction with troponin $\mathrm{T}$. The inhibiting action is eliminated when the conformation of troponin $\mathrm{C}$ changes because of $\mathrm{Ca}^{2+}$ binding to troponin C. This is then followed by the movement of tropomyosin (TPM1) into a non-blocking position that allows myosin to bind to actin.

Thin filament mutations are associated with $5-15 \%$ of HCM cases $[3,4]$. Previous studies demonstrated that mutations in thin filament might result in $\mathrm{Ca}^{2+}$ sensitivity of myofibrillar ATPase activity and altered force generation, which could be responsible for the morphologic change of cardiomyocytes and cardiac dysfunction.

\section{Z-Disc}

The Z-disc, the mechanical integration site of heart and skeletal muscle cells, links the anchorage of myofilaments to force reception and processing. It is probably composed of hundreds of different proteins and is regarded as one of the most complex macromolecular structures in biology. Conventionally, the Z-disc was considered to be the structural unit that protected the sarcomere from damage during contraction. Now, it is also recognized as an important cell-signaling hub.

Since the first recognition of muscle LIM protein (CSRP3) involved in HCM, more mutations in the Z-disc protein have been identified, including telethonin (TCAP), vinculin (VCL), LIM domain binding 3 (LDB3), a-actinin 2 (ACTN2), myozenin-2 (MYOZ2), and cardiac ankyrin repeat protein (ANKRD1). More recently, our laboratory identified another disease gene, NEXN encoding nexilin, which is a crucial protein that functions to protect the cardiac Z-disc from damage that is caused by excessive strain [9]. However, each of these genes is associated with a very small minority of HCM cases.

\section{$\mathrm{Ca}^{2+}$-Handling Proteins}

Intracellular $\mathrm{Ca}^{2+}$ handling is crucial to regulate cardiac contraction and relaxation. The influx of a small amount of $\mathrm{Ca}^{2+}$ into the myocyte triggers a greater release of $\mathrm{Ca}^{2+}$ from the sarcoplasmic reticulum through the ryanodine receptors (RyR2), inducing myocardial contraction. Diastole is initiated by the reuptake of $\mathrm{Ca}^{2+}$ via the sarco/endoplasmic reticulum $\mathrm{Ca}^{2+}$-ATPase (SERCA). The majority of proteins involved in regulating $\mathrm{Ca}^{2+}$ release and reuptake are associated with regu- lating the activity of RyR2 and SERCA. $\mathrm{Ca}^{2+}$ release from the sarcoplasmic reticulum is modulated by accessory proteins such as sorcin (SRI), calstabin (FKBP1B), and calsequestrin (CASQ2). CASQ2 interacts with the luminal sarcoplasmic region of RyR2, promoting an opening in the channel and $\mathrm{Ca}^{2+}$ release. $\mathrm{Ca}^{2+}$ reuptake via the SERCA is reversibly regulated by phospholamban (PLN). Furthermore, some $\mathrm{Ca}^{2+}$ binding/storage chaperones and buffers, such as calreticulin (CALR3) and calmodulin (CALM), are involved in intracellular signaling processes.

Some reports suggested that the genes encoding $\mathrm{Ca}^{2+}$ regulatory proteins, including $P L N, C A S Q 2, C A L R 3$, and junctophilin-2 (JPH2), were implicated in HCM. Mutations in these genes may result in altered $\mathrm{Ca}^{2+}$ handling, contractile dysfunction, and heart failure. In addition, imbalanced $\mathrm{Ca}^{2+}$ homeostasis caused by the $\mathrm{Ca}^{2+}$-handling protein mutations can also induce arrhythmias, energy supply-demand imbalance, cell death, and abnormal transcription regulation. All of these genes are only responsible for relatively rare cases of HCM. Disease-causing genes related to HCM are displayed in table 1.

\section{Relationships between Genotype and Phenotype}

Clinically, it is frequently observed that one causal mutation presents different phenotypes in HCM. This indicates that although HCM is an archetypical single-gene disorder with mendelian inheritance, the phenotypic manifestations are not only determined by causal genes. Other factors (e.g. modifying genes and epigenetic and environmental factors) may also contribute to the complexity of the HCM phenotype.

Despite phenotypic heterogeneity, some correlations between genotype and phenotype are compelling. Generally, MYBPC3 mutations are associated with a later average age at the onset of symptoms, lower incidence of SCD, and a relatively benign clinical course. In contrast, MYH7 mutations often present overt cardiac hypertrophy and a relatively malignant outcome [10]. As for TNNT2 mutations, cardiac hypertrophy is uncommon in children and adolescents, but it is common in adults [11]. Moreover, patients with multiple mutations are commonly diagnosed at an earlier age, develop more overt cardiac hypertrophy, and have a higher risk of SCD than patients with a single mutation [3], even in the absence of any conventional risk factors [12]. These findings suggest that a gene-dosage effect may contribute to the severity of HCM. 
Table 1. Disease-causing genes and the frequency of each gene

\begin{tabular}{|c|c|c|c|c|}
\hline Disease genes & $\begin{array}{l}\text { Evidence for } \\
\text { pathogenicity }\end{array}$ & Locus & Protein & Frequency \\
\hline \multicolumn{5}{|c|}{ Thick filament protein } \\
\hline MYH7 & strong & $14 \mathrm{q} 11.2$ & $\beta$-myosin heavy chain & $15-25 \%$ \\
\hline MYBPC3 & strong & $11 \mathrm{p} 11.2$ & myosin binding protein $\mathrm{C}$ & $15-25 \%$ \\
\hline MYL2 & strong & $12 \mathrm{q} 24.1$ & regulatory myosin light chain & rare \\
\hline MYL3 & strong & $3 \mathrm{p} 21.3$ & essential myosin light chain & rare \\
\hline МYH6 & less & $14 \mathrm{q} 11.2$ & a-myosin heavy chain & rare \\
\hline \multicolumn{5}{|c|}{ Thin filament protein } \\
\hline TNNT2 & strong & $1 q 32.1$ & troponin $\mathrm{T}$ & $\approx 5 \%$ \\
\hline TNNI3 & strong & $11 \mathrm{p} 15.5$ & troponin I & $\approx 5 \%$ \\
\hline TNNC1 & strong & $3 \mathrm{p} 21.1$ & troponin $\mathrm{C}$ & rare \\
\hline TPM1 & strong & $15 \mathrm{q} 22.2$ & a-tropomyosin & rare \\
\hline ACTC1 & strong & $15 \mathrm{q} 14$ & a-cardiac actin 1 & rare \\
\hline \multicolumn{5}{|l|}{ Z-disc protein } \\
\hline CSRP3 & less & $11 \mathrm{p} 15.1$ & muscle LIM protein & rare \\
\hline TCAP & less & $17 q 12$ & telethonin & rare \\
\hline$V C L$ & less & $10 \mathrm{q} 22.2$ & vinculin & rare \\
\hline$L D B 3$ & less & $10 \mathrm{q} 23.2$ & LIM domain binding 3 & rare \\
\hline ACTN2 & strong & $1 \mathrm{q} 43$ & a-actinin 2 & rare \\
\hline MYOZ2 & strong & $4 q 26$ & myozenin 2 & rare \\
\hline ANKRD1 & less & $10 \mathrm{q} 23.3$ & cardiac ankyrin repeat protein & rare \\
\hline NEXN & less & $1 \mathrm{p} 31.1$ & nexilin & rare \\
\hline \multicolumn{5}{|c|}{$\mathrm{Ca}^{2+}$-handling protein } \\
\hline PLN & less & $6 q 22.3$ & phospholamban & rare \\
\hline CASQ2 & less & $1 \mathrm{p} 13.1$ & calsequestrin 2 & rare \\
\hline CALR3 & less & 19 p13.1 & calreticulin 3 & rare \\
\hline JPH2 & less & $20 \mathrm{q} 13.12$ & junctophilin 2 & rare \\
\hline \multicolumn{5}{|c|}{ Other related genes } \\
\hline TTN & less & $2 q 31.2$ & titin & rare \\
\hline CAV3 & less & 3 p25.3 & caveolin 3 & rare \\
\hline
\end{tabular}

In addition, the phenotypic expression of HCM is also affected by modifying genes. Polymorphisms in the genes encoding the renin-angiotensin-aldosterone system, androgen receptors, and calmodulin III may contribute to the phenotypic heterogeneity of HCM [13-15]. More recently, one study about the effect of microRNA on HCM revealed that underproduced microRNA-495 may be associated with cardiac hypertrophy that resulted from a mutation in MYH7 [16].

\section{Pathogenesis}

Currently, it is thought that the potential pathogenesis of HCM involves the following: dominant negative function arising from mutant protein, haploinsufficiency resulting from insufficient normal sarcomere protein, and imbalanced myocardial energetic metabolism.

\section{Dominant Negative Function}

Most of the mutations that are related to HCM are missense alleles producing dominant negative mutant proteins, also referred to as 'poison peptides'. These 'poison peptides' are stable and are incorporated into myofilaments. They affect the normal mechanical functions of sarcomeres and electrophysiological properties of cardiocytes. Mutant proteins may disturb the coordinated contraction of myofilaments. On the other hand, they may increase the actin-activated ATPase activity and force generation by enhancing $\mathrm{Ca}^{2+}$ sensitivity and $\mathrm{Ca}^{2+}$ affinity. The enhanced $\mathrm{Ca}^{2+}$ sensitivity can also bring about the susceptibility to arrhythmia and increased risk of SCD. In addition, mutant proteins are able to stimulate non-myocyte proliferation by stimulating the expression of transforming growth factor- $\beta$. Activated non-myocyte cells secrete profibrotic molecules that increase the stress on mutant cardiomyocytes [17]. The 
persistent activation of this pathway may stimulate the expression of myocyte enhancer factor 2 (MEF-2), resulting in the premature death of myocytes with myocardial scarring, which is a histopathological hallmark of this cardiomyopathy [18].

\section{Haploinsufficiency}

Haploinsufficiency exists when a diploid organism only has a single copy of a gene and does not produce enough products to bring about a wild-type condition. Some mutant products can interfere with the normal mechanical and electrophysiological functions of cardiomyocytes, but other mutant products can be degraded by nonsense-mediated mRNA decay and the ubiquitin-proteasome system [19]. Haploinsufficiency seems to be the primary mechanism for HCM that is caused by $M Y B P C 3$ mutations that are predicted to give rise to premature termination codons and unstable mRNA [20]. Because of the inefficiency of the ubiquitin-proteasome system with age, the degradation of mutant peptides is impaired in older carriers, and a clinical phenotype occurs with the accumulation of abnormal peptides. This, to some extent, can explain why patients with MYBPC3 mutations may present a clinical manifestation at a relatively late age.

\section{Imbalanced Energetic Metabolism}

As a result of the enhanced $\mathrm{Ca}^{2+}$ sensitivity and $\mathrm{Ca}^{2+}$ affinity of mutant proteins, cross-bridge turnover and actin-activated ATPase activity increase. This leads to an apparent gain of protein function at a greater energetic cost of tension generation. In accordance with these biophysical observations, HCM patients without systolic dysfunction displayed functional impairment and morphological disorganization in mitochondria. Energetic abnormalities can be found even in patients carrying the mutation that do not exhibit hypertrophy, which suggests that this change may arise from an abnormity of the sarcomeric function rather than being a result of cardiac hypertrophy. Conversely, the hypertrophy may be a compensatory consequence to the deficiency in energetic metabolism. Therefore, correcting an energetic abnormality may be a potential strategy to improve the clinical manifestation and outcome of HCM. Recently, perhexiline, a drug thought to improve cardiac energetics by shifting substrate use to more efficient carbohydrate metabolism, was shown to correct the diastolic dysfunction and increase the exercise capacity of HCM patients by ameliorating the cardiac energetic impairment [21].

Hypertrophic Cardiomyopathy

\section{Elderly Patients with HCM}

Because the penetrance of HCM is age-dependent (namely the symptoms and signs increase with age), some patients are diagnosed with HCM at a relatively advanced age. The elderly patients present their own clinical features in the distribution of causal genes and phenotypic variation.

\section{Phenotypic Variations}

With respect to the phenotype of HCM, LV hypertrophy is milder in the elderly patients than in the young patients. The LV end-diastolic diameter and left atrial diameter are larger in the elderly patients than in the young patients. Moreover, mitral annular calcification is common in the elderly patients with HCM [22]. The clinical course of HCM can be considered as a lifelong LV remodeling process and subsequent complications. SCD is more common in adolescents and young adults than in elderly patients, but the risk extends across a wide range of ages to mid-life and beyond. On the other hand, with LV remodeling and LV dilation, heart failure was more common in mid-life and beyond [23]. With advancing age, the prevalence of atrial fibrillation increases in HCM patients. The presence of atrial fibrillation was associated with an increased risk of cardiac death, heart failure, and ischemic stroke [24]. Consequently, it is necessary to manage HCM patients from the standpoint of longitudinal evolution in order to prevent these complications.

\section{Distribution of Causal Genes}

HCM in elderly persons is genetically distinct from $\mathrm{HCM}$ in the unselected population. By genetically analyzing 31 US individuals with late-onset HCM (diagnostic age $63 \pm 11$ years), researchers found that 4 individuals harbored mutations in MYBPC3, 2 in TNNI3, and 1 in MYH6 [7]. Another study of 41 Japanese patients with late-onset $\mathrm{HCM}$ (diagnostic age $63 \pm 10$ years) revealed that $4 \mathrm{HCM}$ cases were related to mutations in $M Y B P C 3$ and 1 in $M Y H 7$ [25]. These findings suggest that mutations in MYBPC3 may be the most common cause of delayed expression of HCM. In addition, the sarcomere gene screen was likely to be negative in elderly HCM patients [26]. In a large cohort of $488 \mathrm{HCM}$ probands, DNA was analyzed for mutations in the protein-coding exons of 8 common sarcomeric genes (MYBPC3, MYH7, MYL2, MYL3, TNNT2, TNNI3, TPM1, and ACTC). Of these individuals, only $22 \%$ of patients diagnosed with HCM after the age of 45 years had an identifiable mutation in an 
analyzed sarcomeric gene, whereas $49 \%$ of patients diagnosed before the age of 45 years had a mutation in the same group of analyzed genes.

\section{Clinical Applications of Genetic Testing}

The rapid development of DNA sequencing technology provides opportunities for the identification of pathogenic mutations. However, these mutations can only be identified in $50 \%$ of probands. Moreover, genetic testing often identifies novel DNA sequence variants for which pathogenicity is unresolved. These variants currently have no use for family screening, and they promote confusion in the interpretation of genetic testing results.

At present, the predominant reason for genetic testing in clinical practice is to identify family members who do not express the phenotype but may be at risk of developing disease. This strategy is based on the identification of a pathogenic mutation in the index case. For the relatives without a phenotype who carry a known family mutation, it is necessary to regularly evaluate the changes in their condition. On the contrary, if a phenotype-negative relative carries neither the family mutation nor a de novo mutation, regular evaluation is unnecessary. A negative genetic test not only alleviates their psychological and economic burden of further cardiovascular surveillance but also eliminates restrictions of lifestyle and competitive sports. However, the failure to identify a pathogenic mutation in the proband is an unsure result, which precludes the utility of genetic testing in family members.

Genetic testing can also clarify the diagnosis in patients with metabolic myocardial storage cardiomyopathies, which often present seemingly unexplained myocardial hypertrophy that mimics the HCM phenotype. These disorders are usually referred to as phenocopies and include Fabry disease, an X-linked recessive disease resulting from mutations in the $\alpha$-galactosidase gene (GLA),Danon disease, an X-linked dominant disorder caused by mutations in the lysosome-associated membrane protein gene (LAMP-2), and PRKAG2 cardiac syndrome, an autosomal dominant disorder due to mutations in $5^{\prime}$-AMP-activated protein kinase subunit $\gamma$-2 gene (PRKAG2). Considering the differences in natural history and management strategies, it is of significant importance to distinguish HCM and its phenocopies. However, sometimes these phenocopies pose dilemmas for the clinicians making the correct diagnosis. Now, genetic testing can be used as an additional approach to assist the clinicians in making the diagnosis.

\section{Conclusion}

In spite of the great progress made towards the identification of genetic causes and pathogenesis of HCM in the past two decades, there are still more questions that need to be better understood about this mendelian disorder. It remains a great challenge to ascertain the cause of $50 \%$ of HCM cases without identified mutations. The failure to identify a mutation may be a false negative due to incomplete genetic investigation. The application of genomewide association studies technology in these patients may uncover the genetic background of these cases. In addition, although the pathogenesis of HCM has been uncovered to some extent, the impact of a causal mutation on the function and signaling of cardiocytes is expected to be elucidated further. Only by comprehensively understanding the detailed mechanisms can new therapeutic targets be found successfully in the not-too-distant future.

\section{Acknowledgments}

We thank for Hu Wang and Jizheng Wang for their suggestions about this work. This work was funded by the Ministry of Science and Technology of China (grant Nos. 2009DFB30050 and 2010CB732601) and the National Natural Science Foundation of China (grant No. 30971233).

References

- 1 Force T, Bonow RO, Houser SR, Solaro RJ, Hershberger RE, Adhikari B, Anderson ME, Boineau R, Byrne BJ, Cappola TP, Kalluri R, LeWinter MM, Maron MS, Molkentin JD, Ommen SR, Regnier M, Tang WH, Tian R, Konstam MA, Maron BJ, Seidman CE: Research priorities in hypertrophic cardiomyopathy: report of a Working Group of the National Heart, Lung, and Blood Institute. Circulation 2010;122:1130-1133.

2 Maron BJ, Maron MS, Semsarian C: Genetics of hypertrophic cardiomyopathy after 20 years: clinical perspectives. J Am Coll Cardiol 2012;60:705-715.

-3 Olivotto I, Girolami F, Ackerman MJ, Nistri S, Bos JM, Zachara E, Ommen SR, Theis JL, Vaubel RA, Re F, Armentano C, Poggesi C, Torricelli F, Cecchi F: Myofilament protein gene mutation screening and outcome of patients with hypertrophic cardiomyopathy. Mayo Clin Proc 2008;83:630-638.

4 Millat G, Bouvagnet P, Chevalier P, Dauphin C, Jouk PS, Da Costa A, Prieur F, Bresson JL, Faivre L, Eicher JC, Chassaing N, Crehalet H, Porcher R, Rodriguez-Lafrasse C, Rousson $\mathrm{R}$ : Prevalence and spectrum of mutations in a cohort of 192 unrelated patients with hypertrophic cardiomyopathy. Eur J Med Genet 2010;53:261-267. 
5 Maron MS, Olivotto I, Zenovich AG, Link MS, Pandian NG, Kuvin JT, Nistri S, Cecchi F, Udelson JE, Maron BJ: Hypertrophic cardiomyopathy is predominantly a disease of left ventricular outflow tract obstruction. Circulation 2006;114:2232-2239.

-6 Gersh BJ, Maron BJ, Bonow RO, Dearani JA, Fifer MA, Link MS, Naidu SS, Nishimura RA, Ommen SR, Rakowski H, Seidman CE, Towbin JA, Udelson JE, Yancy CW: 2011 ACCF/ AHA guideline for the diagnosis and treatment of hypertrophic cardiomyopathy: executive summary: A report of the American College of Cardiology Foundation/American Heart Association Task Force on Practice Guidelines. Circulation 2011;124:2761-2796.

7 Niimura H, Patton KK, McKenna WJ, Soults J, Maron BJ, Seidman JG, Seidman CE: Sarcomere protein gene mutations in hypertrophic cardiomyopathy of the elderly. Circulation 2002;105:446-451.

8 Walsh R, Rutland C, Thomas R, Loughna S: Cardiomyopathy: a systematic review of disease-causing mutations in myosin heavy chain-7 and their phenotypic manifestations. Cardiology 2010;115:49-60.

-9 Wang H, Li Z, Wang J, Sun K, Cui Q, Song L, Zou Y, Wang X, Liu X, Hui R, Fan Y: Mutations in NEXN, a Z-disc gene, are associated with hypertrophic cardiomyopathy. Am J Hum Genet 2010;87:687-693.

$\checkmark 10$ Wang S, Zou Y, Fu C, Xu X, Wang J, Song L, Wang H, Chen J, Huan T, Hui R: Worse prognosis with gene mutations of $\beta$-myosin heavy chain than myosin-binding protein $\mathrm{C}$ in Chinese patients with hypertrophic cardiomyopathy. Clin Cardiol 2008;31:114-118.

-11 Pasquale F, Syrris P, Kaski JP, Mogensen J, McKenna WJ, Elliott P: Long-term outcomes in hypertrophic cardiomyopathy caused by mutations in the cardiac troponin $\mathrm{T}$ gene. Circ Cardiovasc Genet 2012;5:10-17.

12 Maron BJ, Maron MS, Semsarian C: Double or compound sarcomere mutations in hypertrophic cardiomyopathy: a potential link to sudden death in the absence of conventional risk factors. Heart Rhythm 2012;9:57-63.
13 Van der Merwe L, Cloete R, Revera M, Heradien M, Goosen A, Corfield VA, Brink PA, Moolman-Smook JC: Genetic variation in angiotensin-converting enzyme- 2 gene is associated with extent of left ventricular hypertrophy in hypertrophic cardiomyopathy. Hum Genet 2008;124:57-61.

14 Landstrom AP, Parvatiyar MS, Pinto JR, Marquardt ML, Bos JM, Tester DJ, Ommen SR, Potter JD, Ackerman MJ: Molecular and functional characterization of novel hypertrophic cardiomyopathy susceptibility mutations in TNNC1-encoded troponin C. J Mol Cell Cardiol 2008;45:281-288.

15 Friedrich FW, Bausero P, Sun Y, Treszl A, Kramer E, Juhr D, Richard P, Wegscheider K, Schwartz K, Brito D, Arbustini E, Waldenstrom A, Isnard R, Komajda M, Eschenhagen T, Carrier L: A new polymorphism in human calmodulin III gene promoter is a potential modifier gene for familial hypertrophic cardiomyopathy. Eur Heart J 2009;30:16481655.

16 Palacin M, Reguero JR, Martin M, Diaz Molina B, Moris C, Alvarez V, Coto E: Profile of micrornas differentially produced in hearts from patients with hypertrophic cardiomyopathy and sarcomeric mutations. Clin Chem 2011;57:1614-1616.

17 Teekakirikul P, Eminaga S, Toka O, Alcalai $\mathrm{R}$, Wang L, Wakimoto H, Nayor M, Konno T, Gorham JM, Wolf CM, Kim JB, Schmitt JP, Molkentin JD, Norris RA, Tager AM, Hoffman SR, Markwald RR, Seidman CE, Seidman JG: Cardiac fibrosis in mice with hypertrophic cardiomyopathy is mediated by non-myocyte proliferation and requires TGF- $\beta$. J Clin Invest 2010;120:3520-3529.

18 Konno T, Chen D, Wang L, Wakimoto H, Teekakirikul P, Nayor M, Kawana M, Eminaga S, Gorham JM, Pandya K, Smithies O, Naya FJ, Olson EN, Seidman JG, Seidman $\mathrm{CE}$ : Heterogeneous myocyte enhancer factor-2 (MEF-2) activation in myocytes predicts focal scarring in hypertrophic cardiomyopathy. Proc Natl Acad Sci USA 2010;107: 18097-18102.

19 Vignier N, Schlossarek S, Fraysse B, Mearini G, Kramer E, Pointu H, Mougenot N, Guiard J, Reimer R, Hohenberg H, Schwartz K, Vernet M, Eschenhagen T, Carrier L: Nonsense-mediated MRNA decay and ubiquitinproteasome system regulate cardiac myosinbinding protein $\mathrm{C}$ mutant levels in cardiomyopathic mice. Circ Res 2009;105:239-248.
20 Marston S, Copeland O, Jacques A, Livesey K, Tsang V, McKenna WJ, Jalilzadeh S, Carballo S, Redwood C, Watkins H: Evidence from human myectomy samples that MYBPC3 mutations cause hypertrophic cardiomyopathy through haploinsufficiency. Circ Res 2009;105:219-222.

21 Abozguia K, Elliott P, McKenna W, Phan TT, Nallur-Shivu G, Ahmed I, Maher AR, Kaur $\mathrm{K}$, Taylor J, Henning A, Ashrafian H, Watkins H, Frenneaux M: Metabolic modulator perhexiline corrects energy deficiency and improves exercise capacity in symptomatic hypertrophic cardiomyopathy. Circulation 2010;122:1562-1569.

22 Kubo T, Kitaoka H, Okawa M, Matsumura Y, Hitomi N, Yamasaki N, Furuno T, Takata J, Nishinaga M, Kimura A, Doi YL: Lifelong left ventricular remodeling of hypertrophic cardiomyopathy caused by a founder frameshift deletion mutation in the cardiac myosin-binding protein $\mathrm{C}$ gene among Japanese. J Am Coll Cardiol 2005;46:1737-1743.

23 Kitaoka H, Kubo T, Okawa M, Hitomi N, Furuno T, Doi YL: Left ventricular remodeling of hypertrophic cardiomyopathy: longitudinal observation in rural community. Circ J 2006;70:1543-1549.

24 Olivotto I, Cecchi F, Casey SA, Dolara A, Traverse JH, Maron BJ: Impact of atrial fibrillation on the clinical course of hypertrophic cardiomyopathy. Circulation 2001;104: 2517-2524.

-25 Anan R, Niimura H, Takenaka T, Hamasaki S, Tei C: Mutations in the genes for sarcomeric proteins in Japanese patients with onset sporadic hypertrophic cardiomyopathy after age 40 years. Am J Cardiol 2007;99: 1750-1754.

26 Binder J, Ommen SR, Gersh BJ, Van Driest SL, Tajik AJ, Nishimura RA, Ackerman MJ: Echocardiography-guided genetic testing in hypertrophic cardiomyopathy: septal morphological features predict the presence of myofilament mutations. Mayo Clin Proc 2006;81:459-467. 